\title{
Why have a special issue on methods used in clinical pharmacy practice research?
}

\author{
Timothy F. Chen ${ }^{1}$ Carmel M. Hughes ${ }^{2}$
}

Received: 14 May 2016/Accepted: 20 May 2016/Published online: 4 June 2016

(c) Springer International Publishing 2016

This Special Issue of the Journal focuses on methodologies which are particularly pertinent to practice and clinical research in the field of pharmacy. Our readers may ask why such an issue has to be published. Research in our discipline is evolving, and the demand for evidence to inform practice and policy has never been greater. The generation of that evidence needs to be grounded in rigorous and robust methodologies, which are used appropriately. Increasingly sophisticated methods of analysis are being developed and employed, and researchers need to know how these analytical approaches should be applied and interpreted. The division between quantitative and qualitative approaches is no longer clear-cut, and mixed methods are seen as entirely appropriate in certain contexts. In an effort to improve transparency and quality, researchers are expected to follow checklists when reporting on particular types of studies e.g. the CONSORT guidelines for randomised controlled trials (RCTs).

It is true that many of the research methods used in clinical pharmacy and pharmacy practice research have been "borrowed" and "adapted" from related fields including psychology, sociology, clinical medicine, business and economics. Overall, this has led to a greater emphasis on the appropriate choice of a specific method in order to answer a specific question. As a consequence,

Special edition guest editors.

Timothy F. Chen

timothy.chen@sydney.edu.au

1 Faculty of Pharmacy, The University of Sydney, Camperdown, NSW 2006, Australia

2 School of Pharmacy, Queen's University Belfast, 97 Lisburn Road, Belfast, BT9 7BL, Northern Ireland, UK researchers must now place a stronger emphasis on the precise interpretation of their results, the testing of specific hypotheses, the internal validity of their data and the reliability of the methods used, amongst other considerations. Increasingly there will be a need to acknowledge methodological limitations (and strengths) if the discipline of clinical pharmacy research is to be considered an "equal" amongst other fields. Equal in the sense that it will be recognised by others as a true discipline, able to compete and succeed in gaining competitive funding and be published in journals of the highest calibre, in addition to our own, highly regarded, discipline-specific journals.

In light of these developments, we hope that the papers published in this Special Issue will stimulate and assist those who engage in pharmacy research. Readers will note the range of topics which are covered, starting with literature reviews which are often the basis for a research project, the use of theory in research, and future methods in pharmacy practice research. A range of qualitative methods are highlighted such as narrative interviewing, grounded theory methods, and nominal group techniques. The growing importance of the appropriate use of mixed methods is discussed, along with the core quantitative approaches such as quasi-experimental designs and prospective cluster randomised controlled intervention trials. As governments across the world face increasing economic challenges, high quality research using the most appropriate methodologies will help inform decisionmaking at policy level, and ensure that effective and costeffective services are delivered which will improve health outcomes at a patient and population level.

We think the signs regarding methodological quality improvement in pharmacy practice research are very good! Many researchers in pharmacy practice have published high quality systematic reviews, including meta-analyses, 
(e.g. The Cochrane Collaboration) and meta-syntheses, producing what is often considered the highest level of evidence. There are many highly cited randomised controlled studies of medication management and other pharmaceutical care interventions. Quasi-experimental designs have been employed when randomisation has not been appropriate or possible when implementing complex interventions. Cross-sectional studies have used survey instruments with sound psychometric properties. Qualitative studies have employed sophisticated methods to determine rich and meaningful data with high internal validity, based on established theoretical frameworks or building new theories about medicine use. These robust methods, amongst many others, have often been coupled with sophisticated multivariate analyses and higher level conceptual interpretations which have enabled the answering of specific and complex research questions pertaining to the practice of pharmacy. The fact that there are now numerous researchers in pharmacy who are recognised as experts in the use of specific methods is a critical phase in our development as a discipline. Although we believe that early career researchers should have a firm grounding in a variety of research methods which can be used in pharmacy practice, having experts in the use of particular methods is crucial to the advancement of our discipline.

We hope that readers will enjoy this Special Issue. Our goal was to gather, in one issue of the Journal, an overview and critical insight into some key methodological approaches which may be applied to clinical pharmacy and pharmacy practice research. We understand that not all approaches have been covered-this is always a challenge when space is limited. Nevertheless, we hope that these articles might be useful for both early career and more experienced researchers alike. Happy reading! 